Published in final edited form as:

Trends Biotechnol. 2011 May ; 29(5): 222-230. doi:10.1016/j.tibtech.2011.01.003.

\title{
Measuring enzyme activity in single cells
}

\author{
Michelle L. Kovarik ${ }^{1}$ and Nancy L. Allbritton ${ }^{1,2}$ \\ ${ }^{1}$ Department of Chemistry, University of North Carolina, Chapel Hill, North Carolina 27599 \\ ${ }^{2}$ Department of Biomedical Engineering, University of North Carolina, Chapel Hill, NC 27599 and \\ North Carolina State University, Raleigh, NC 27695
}

\begin{abstract}
Seemingly identical cells can differ in their biochemical state, function and fate, and this variability plays an increasingly recognized role in organism-level outcomes. Cellular heterogeneity arises in part from variation in enzyme activity, which results from interplay between biological noise and multiple cellular processes. As a result, single-cell assays of enzyme activity, particularly those that measure product formation directly, are crucial. Recent innovations have yielded a range of techniques to obtain these data, including image-, flow- and separationbased assays. Research to date has focused on easy-to-measure glycosylases and clinicallyrelevant kinases. Expansion of these techniques to a wider range and larger number of enzymes will answer contemporary questions in proteomics and glycomics, specifically with respect to biological noise and cellular heterogeneity.
\end{abstract}

\section{The role of enzyme activity in cellular heterogeneity}

Biochemical measurements of single cells are becoming more prevalent as appreciation grows for heterogeneity among seemingly identical cells [1-3]. Cellular heterogeneity is now recognized to have substantial impact at the organism level on processes such as embryonic development [4], immune response [5-6], efficacy of chemotherapy [7-8], and evolutionary fitness [3]. These macroscopic effects of cellular heterogeneity arise from variability in the outputs of complex biochemical pathways that regulate cell function. For example, a population of cells might respond heterogeneously to a homogeneous stimulus (Figure 1a,b). Underlying such variability are differences in individual components of the cell's response pathway, many of which involve enzymatic reactions. Single-cell assays of enzyme activities provide detailed information about variability in the individual biochemical steps that affect downstream outcomes (Figure 1c,d), and these measurements are therefore integral to elucidating the biochemical origins of cellular heterogeneity.

Classical enzymology techniques typically use tissue homogenates or purified proteins to study enzyme activity as a function of time for known enzyme concentrations, but new approaches are needed to follow enzymatic reactions in individual cells. Fortuitously, recent

\footnotetext{
(C) 2011 Elsevier Ltd. All rights reserved.

Corresponding author: Allbritton, N.L. (nlallbri@unc.edu)..

Publisher's Disclaimer: This is a PDF file of an unedited manuscript that has been accepted for publication. As a service to our customers we are providing this early version of the manuscript. The manuscript will undergo copyediting, typesetting, and review of the resulting proof before it is published in its final citable form. Please note that during the production process errors may be discovered which could affect the content, and all legal disclaimers that apply to the journal pertain.

Conflict of interest statement

NLA owns less than $1 \%$ of the stock of Cell Biosciences (Palo Alto, CA), which holds license to patents concerning the technologies described in [56] and [57].
} 
research has led to a convergence of scientific interest in biological heterogeneity and the technological capabilities required to analyze single cells, resulting in rapid progress in this area. We discuss the origins of heterogeneity in enzyme activity; summarize recent breakthroughs in single-cell techniques based on imaging, flow-through systems, and electrophoretic separations; and conclude by highlighting promising areas for future research in single-cell analysis.

\section{Origins of cellular heterogeneity in enzyme activity}

Cellular heterogeneity is complex in origin, and, particularly in the case of enzyme activity, derives from multiple biological processes. At the nucleic acid level, heterogeneity arises through genetic mutations, epigenetic modifications, and transcriptional regulation (Figure 2a-c). At the protein level, translational regulation, post-translational modifications, and protein degradation (Figure 2d-f) contribute to differential enzyme activity between cells. These processes affect enzyme activity primarily by changing either the chemical identity of the enzyme (e.g. by genetic mutations, transcriptional or translational errors, or posttranslational modification) or by changing the enzyme concentration (e.g. by regulation of transcription, translation or degradation). All of these processes working in concert produce the observed biological output, and variation in each process contributes to cellular heterogeneity [3].

Cell-to-cell variation in these biological processes exists in part because of functionaldifferences between cells, such as level of differentiation; but variability also arises in otherwise homogeneous cell populations owing to biological noise. Biological noise refers to the inherent variability between otherwise identical cells and can be intrinsic or extrinsic [9]. Intrinsic noise arises from stochastic fluctuations in biochemical events, such as binding of an enzyme to its substrate, whereas extrinsic noise is correlated to a physical parameter, such as microenvironment, cell cycle stage, or even intrinsic noise in an upstream event [10-11]. Although biological noise is an inevitable consequence of the stochastic nature of some cellular events, recent work indicates that biological noise might also be a functional component of specific biological processes [12-13]. For example, studies in the yeast Saccharomyces cerevisiae have shown that proteins involved in stress response exhibit higher than expected noise levels, possibly because the population as a whole benefits from a more diverse response to stressful environmental conditions [12]. To date, most research on biological noise has examined mRNA or protein copy number $[10,12,14-15]$ rather than variability in enzyme activity. As technology for single-cell measurements matures, direct measures of noise in enzyme function will be possible. Consequently, depending on the goals and design of a given study, biological noise might be a peripheral consideration addressed during statistical analyses or, conversely, a major focus of the work [1].

Because enzymatic activity in an individual cell depends on a dynamic interplay between biological noise and biochemical regulation, direct assays of enzyme activity (i.e. those that measure conversion of substrate into product) are crucial. Until recently, these assays were difficult to perform at the single-cell level, and a much larger body of research on single cells has used proxy measurements, such as confirming the presence of enzyme or phosphoenzyme via immunostaining or gene expression studies. These experiments provide large quantities of valuable information about cellular heterogeneity and its biomedical consequences (for example, see [16]), but the presence of enzyme or phospho-enzyme alone does not necessarily correspond to enzyme activity. As a result, direct assays of enzyme activity contribute to a more complete picture of cellular heterogeneity. Activity measurements further complement other single-cell methods in their ability to assay low abundance enzymes. Low copy number enzymes are below the detection limit of many 
measurement technologies, but their activities may be quantifiable without specialized equipment because a single enzyme turns over numerous substrate molecules, effectively amplifying the signal [17].

An ideal single-cell enzyme assay would measure the activities of multiple enzymes with high spatiotemporal resolution and high throughput. In reality, no single technique is likely to achieve all of these goals; as a result, a wide range of complementary methods and technologies are currently being developed. The following sections examine three broad categories of single-cell enzyme assays: those based on imaging, flow-through systems, and separations, and combinations thereof.

\section{Image-based analyses}

Microscopy might be considered the original single-cell analytical tool. Variations in size, morphology, and biochemical state and response to stimuli (with an appropriate indicator) are all readily examined with high spatiotemporal resolution. Indeed, some of the earliest single-cell enzyme assays used microscopy in combination with fluorogenic substrates [18], and many fluorogenic substrates are commercially available [19]. Additionally, substrates based on fluorescence-resonance energy transfer (FRET) are now widely used to assay proteases and kinases (see Glossary) [20-21]. (FRET sensors for GTPases are also available, but report on whether the enzyme itself is in its active or inactive form [22].) Unlike fluorogenic substrates, many FRET substrates undergo reversible conformation changes so the resulting signal reflects the activities of competing enzymes, such as kinases and phosphatases. Recently, two substrates containing different FRET pairs have been applied to simultaneous assays of pro-tumorigenic Src kinase and a matrix metalloprotease (Figure 3a), revealing important temporal clues about the relationship between the two enzymes [23]. In addition to fluorogenic and FRET substrates, several alternative fluorescence-based reagents have been developed for kinases and proteases. For kinase assays, peptide-based sensors respond to changes in fluorophore environment or proximity to a quencher [24]. Novel protease assays rely on diffusion of a cleaved reporter from the evanescent field of a nanoprobe [25], uptake of fluorescent or fluorogenic materials [26-27], or translocation of a fluorescent protein to the nucleus [28].

Microscopy-based analyses are also profiting from improved methods for 3D confinement of individual cells or single-cell lysates. Entrapment of nonadherent cells benefits time-lapse studies by immobilizing each cell, whereas confinement prevents excessive dilution and mixing of single-cell lysates. A variety of methods can be used to isolate intact cells, including liquid walls formed by immiscible fluids and solid walls produced by microfabrication. Early analyses used an oil layer to confine single cell lysates to individual nanoliter-volume droplets [29]. Recently, microchannel-based fluid handling has dramatically increased throughput and decreased the confinement volumes achievable with this strategy; individual cells can now be sorted into picoliter-volume droplets, lysed in the presence of a fluorogenic substrate, and assayed for enzymatic activity [30]. Additionally, the microfluidic platform used for droplet formation is amenable to high-throughput, flowbased readout of the fluorescence signal in addition to more traditional image-based detection [31].

Microfabricated traps and wells are alternative means of cell isolation that provide partial or complete confinement using solid structures. Rapid advances in microfabrication have led to techniques that produce structures with length scales comparable to the size of eukaryotic cells (microns) [32]. As a result, laboratories developing novel fabrication techniques routinely produce sophisticated architectures for cell-based assays [33], and even nonspecialists have access to simpler technologies, including chemical etching and soft 
lithography. One strategy traps cells by flowing them through arrays of microfabricated obstacles. Captured cells are held in place during wash steps and reagent additions, allowing kinetic information to be obtained from individual cells [34]. Alternatively, microwells isolate individual cells in arrays of cell-sized holes, and integrated fluidic components allow for the addition of lysis reagents, fluorogenic substrates, and inhibitors (Figure 3b) [35]. Similar microwells can be used with electrochemical detection. In these cases, electroactive product from lysed cells is confined in the microwells and detected by scanning a microelectrode over the array [36]. Scanning electrochemical microscopy (SECM) can also be used to image single-cell enzyme activity in the absence of microarrays (Figure 3c) [37]. In this work, confinement is achieved by leaving the cell membrane intact and using digitonin to perforate the membrane, thus allowing substrate and product to diffuse in and out of the cell. An image is produced by scanning an ultramicroelectrode over the cell surface and measuring current at each pixel [37].

While SECM represents a more exotic image-based method, most of the assays described in this section require only a fluorescence microscope and appropriate reagents. As a result, image-based methods are widely used, particularly in laboratories that do not specialize in single-cell studies. Image-based techniques are usually non-destructive, allowing multiple measurements to be made on the same cell over time and providing useful kinetic information. A wide range of fluorogenic, FRET, and other substrates are available for enzyme assays; however, the number of indicators is still dwarfed by the number of enzymes expressed in a living cell. Empirical guidelines for indicator design exist [38], but validation and optimization of new indicators and substrates are challenging and timeconsuming, particularly because probes do not always respond to chemical or environmental changes in a predictable manner [39-40]. Additionally, despite advances in automated scanning cytometers, most image-based methods are relatively low-throughput compared to flow cytometry, and spectral overlap limits the number of enzymes that can be probed simultaneously. These limitations are addressed by flow- and separation-based techniques, respectively, as discussed below.

\section{Flow-based methods}

While image-based analyses excel at providing spatiotemporal information about enzyme activity, flow-based methods often trade these details for increased throughput. Indeed, flow cytometry represents the gold standard for high-throughput single-cell measurements. Flow cytometric analyses often use fluorescent antibodies to measure enzyme (or phosphoenzyme) concentration as a proxy for enzyme activity, but some studies measure production of a fluorescent product instead [41-42]. Many fluorogenic substrates used in imaging and fluorimetry studies have also been applied in a flow cytometry format [43-46]. The highthroughput achieved allows representative populations of cells to be examined in reasonable time frames, which is a major benefit, particularly in clinical applications. For example, flow cytometry has been used to study Gaucher's disease, which is characterized by glucocerebrosidase deficiency, in patient samples (Figure 4a) [47]. Flow-through assays can also be performed with amperometric detection of electroactive products from perforated cells; to date, glucose-6-phosphate dehydrogenase [17] and peroxidase [48] have been assayed in this format. Finally, imaging flow cytometers, such as the ImageStream ${ }^{x}$ from Amnis Corp., combine flow cytometry with microscopy to obtain spatial information and morphology along with traditional flow cytometry data.

\section{Separation-based methods}

Capillary electrophoresis (CE) combined with laser-induced fluorescence (LIF) is the separation method of choice in single-cell enzyme assays because of its capacity for rapid, 
high-efficiency separations, its compatibility with picoliter-volume samples, and its exquisitely sensitive detection limits. The peak capacity of even a modest CE separation ( $n$ $\approx 50-100$ ) [49] exceeds the number of fluorescent dyes that can be spectrally resolved ( $\mathrm{n}<$ 20) [50]. Relatively simple LIF systems provide detection limits of $10^{-20}$ to $10^{-21}$ moles, and data analysis for multiple analytes is simpler than for multi-color systems. Separation-based assays are therefore particularly well-suited for the analysis of multiple enzymes in a single cell.

Two strategies can be employed in separation-based assays: (i) separation of multiple enzymes followed by enzymatic reaction; or (ii) enzymatic reaction followed by separation of unreacted substrate and product(s). In the first method, enzymes from a lysed cell are electrophoretically separated in a run buffer containing the enzyme substrate. After preliminary separation, the high voltage is removed, and an incubation period allows each enzyme to convert nearby substrate molecules into product. High voltage is then reapplied to carry the product zones to the detector. This technique is particularly well-suited to studies of closely related enzymes for which individually specific substrates may not be available. For example, isoforms of lactate dehydrogenase from a single red blood cell have been electrophoretically separated, and the resulting zones of nicotinamide adenine dinucleotide (NADH) detected by fluorescence [51]. More recently, the same strategy has been used with electrochemical detection [52]. In this case, two isoforms of alkaline phosphatase were separated and their relative activities determined via amperometric detection of phenol production.

The second method uses CE for multi-enzyme measurements based on separation of unreacted substrate(s) from product(s). For these studies, a reporter substrate is loaded into the cell and the enzymatic reaction proceeds within the intact cell. As a result, competing enzymes act on the substrate in concert, and the resulting substrate to product ratio reflects the net enzymatic activity from these reactions. After reporter loading and incubation, each cell is lysed [53] or sampled with a capillary [54-55], and its contents are separated and detected. This method has been used with several peptide substrates [56] and was recently applied to a lipid reporter for phosphoinositide 3-kinase (PI3K) (Figure 4b) [57]. An advantage of this method is that it reflects the in vivo enzyme state where proteins often compete for activating or inhibiting binding partners and are regulated by the concentration of small molecules such as ions and second messengers. Additionally, separation of cell contents prior to detection allows both unreacted substrate and products to be quantified. This is important because the amount of product formed depends upon the concentration of substrate loaded (Figure 1d), and assays that measure only product formation might be confounded by cell-to-cell heterogeneity in substrate uptake. Separation-based methods account for this variability by permitting ratiometric analysis of substrate and product signals and quantification of the number of moles of each per cell.

Multi-analyte detection and measurement of complete signaling cascades are critical to understanding how the activities of enzymes co-vary and how regulation and feedback mechanisms work in individual cells. Separation-based analyses eliminate the need for detector specificity for each analyte and therefore increase the number of analytes that can be detected from each cell. To date, we have assayed the activities of up to five enzymes simultaneously from a single cell (N. Allbritton, unpublished). This method is also useful in cases where several enzymes may act on a single substrate sequentially, resulting in multiple product species [58-59]. The primary challenges to expanded multi-analyte assays are the need for thoughtful reporter design, effective means of substrate delivery to intact cells, and increased throughput. Effective reporters must have a known specificity and be resistant to degradation by peptidases and proteases (unless these are the enzymes of interest, as in Figure 1d). Reporters must also be effectively loaded into individual cells by physical (e.g. 
microinjection [56]), chemical (e.g. myristoylation [60]), or biological (e.g. endogenous production [61] or cellular uptake [58-59]) means. Finally, while separation-based methods have not yet achieved the throughput of flow cytometry, increased automation [62-63] and the development of microfluidic platforms for these assays [64-65] promise rapid progress in this area in the near future.

\section{Future prospects}

The technologies described above continue to be refined and validated by comparison to more established ensemble-averaging techniques. As these methods mature, they must be applied to biologically interesting problems. To date, most publications on single-cell enzyme assays demonstrate the feasibility and performance of a new technique using a single, well-characterized enzyme with a readily available substrate (e.g. $\beta$-galactosidase with engineered fluorogenic substrates [18,30,54-55]). While these proof-of-principle studies are necessary steps in assay development, they should be followed up with novel applications or clinical samples as quickly as possible. High-impact publications, in which the data rather than the methodology provide the story, encourage broader adoption of new techniques and highlight the importance of single-cell analyses in the scientific community at large.

The development of single-cell assays for a wider range of enzymes is critical to this endeavor. In Figure 5a, the enzymes for which single-cell assays have been published are grouped by their top-level Enzyme Commission (EC) number. EC numbers specify the chemical reaction catalyzed by an enzyme, with each level of the EC number more finely defining the reaction involved. For comparison, the distribution of human enzymes listed in the Braunschweig Enzyme Database (BRENDA: www.brenda-enzymes.org) is also given (Figure 5b) [66]. Comparison of these plots illustrates two important points about current single-cell enzyme assays. First, the number of enzymatic reactions for which single-cell assays have been demonstrated is small $(n=42)$ compared to the total diversity of enzymecatalyzed reactions in humans $(\mathrm{n}=2565)$. As a result, the field is wide open for development and application of assays for uncharacterized enzymes from any class. Secondly, we note that oxidoreductases (EC 1), lyases (EC 4) and isomerases (EC 5) are underrepresented, while transferases (EC 2) and hydrolases (EC 3) are overrepresented.

Transferases (EC 2) are well-represented in single-cell assays because this category includes kinases, an important enzyme class implicated in cancers and other disease states. In contrast, hydrolases are overrepresented simply because they catalyze reactions that are readily assayed by traditional single-cell technologies. The distribution of hydrolases with published single-cell assays is broken out to the second EC level in Figure 5c, and the same data are shown for all human hydrolases in BRENDA in Figure 5d. Glycosylases (EC 3.2), which include $\beta$-galactosidase and $\alpha$-glucosidase, are dramatically overrepresented in the single-cell assays because fluorogenic substrates for these enzymes have been readily available and well-characterized for decades. In contrast, many biologically interesting problems require substrates that undergo multiple reactions catalyzed by a variety of enzymes. For example, oncogenic Ras proteins undergo several post-translational modifications (PTMs), including farnesylation, endoproteolysis, and carboxyl-methylation, before becoming functional. A fluorescent reporter peptide has been developed to assay these reactions, but the challenges of effective substrate design are underscored by the fact that, to date, the reported in vivo products differ from products predicted from in vitro reactions [67].

Expanding single-cell enzyme assays to a wider range of enzymes will allow several broadly important questions in contemporary biological research to be answered at the level of 
individual cells. Current research in popular "omics" fields is now moving beyond the templated processes of genomics and protein translation and into the enzymatically controlled arenas of PTMs, glycomics, and metabolomics. The proteomics field bridges templated translation and enzymatic PTMs; and, as the field matures, increased emphasis is being placed on PTMs [68] and single-cell analyses [69]. Single-cell assays for the enzymatic reactions that control PTMs will complement existing mass spectrometric techniques, which are more commonly applied to ensemble measurements. For example, glycosylation is a specific PTM involving enzymatic addition of sugar molecules to a protein (or lipid). The resulting complex polysaccharides act as structural elements in the cell, facilitate protein-protein interactions, and function as endogenous and exogenous recognition molecules [70]. A coordinated suite of enzymes control the synthesis and metabolism of these molecules, and single-cell assays to probe these reactions would be a major contribution to glycobiology. Single-cell analysis will also contribute to the emerging field of metabolomics. For example, cancer cells are metabolically distinct from normal cells because the enzyme network that executes glycolysis and lactic acid fermentation is favored over the citric acid cycle, which results in high rates of lactate formation. This effect has recently been observed at the single-cell level using a nanoprobe [71].

Finally, increased throughput and larger sample sizes will allow single-cell enzyme assays to contribute to the growing knowledgebase on biological noise. Recent research on biological noise maps variation in transcription and translation [11-12,15], raising the questions of how or whether fluctuating protein levels are reflected in enzyme activity. Cells exercise posttranslational control over enzyme activities through chemical modifications, such as phosphorylation, and sub-cellular localization; however, the extent to which these regulatory processes temper noise in protein levels is still to be determined. Paired measurements of enzyme concentration and activity at the single-cell level promise to address this question. Whatever the results of these experiments, the outlook for continued contributions from single-cell enzyme assays to biology, medicine and biotechnology is bright.

\section{Box 1. Biological insights from single-cell enzyme assays}

Single-cell enzyme assays provide unique spatial and temporal information and identify subpopulations that are obscured by ensemble measurements. Several biological insights obtained by single-cell enzyme assays are highlighted below.

\section{Spatial information}

Bulk measurements are unable to pinpoint enzymatic activity to specific cellular structures or locations, but image-based single-cell (or single-organelle) assays can. For example, protein kinase A (PKA) activity is regulated in part by localization of the active kinase to specific regions of the cell. A suitable indicator combined with microscopic observation of intact mitochondria distinguishes the relative activity of PKA in three microenvironments: the outer membrane, the intermembrane space, and the matrix [72].

\section{Temporal information}

When cellular heterogeneity causes cellular responses to vary in time, single-cell measurements clarify the relative timing of events. For example, cells may tolerate an apoptotic stimulus (e.g. tumor necrosis factor- $\alpha$, staurosporine, etoposide) for hours before a sudden, rapid increase in caspase-3 activity leads to cell lysis [73]. Ensemble measurements of the same enzyme activity show a slow and steady increase uncharacteristic of any actual cell's response. Similarly, cells treated with epithelial growth factor (EGF) exhibit a rapid rise in Src kinase activity. Simultaneous measurements of membrane type 1 matrix metalloprotease (MT1-MMP) in individual cells show that the spike in Src kinase activity is correlated with a gradual rise in MT1- 
MMP activity [23]. Finally, single-cell analyses can also detect heterogeneity in enzyme activity owing to time-linked variations in cell cycle stage [58] or cell age [51].

\section{Identification of subpopulations}

Single-cell enzyme assays also expose cellular heterogeneity, non-Gaussian population distributions, and subpopulations that are averaged out of ensemble measurements. For example, individual neurons differ in their metabolism of gangliosides, which are important membrane components implicated in several genetic disorders. Single-cell assays with fluorescently tagged ganglioside $\mathrm{G}_{\mathrm{M} 1}$ have been able to probe this cellular heterogeneity in lipid metabolism [74]. Similarly, drug-resistant subpopulations might be obscured by bulk measurements, but are readily detected in single-cell assays. Lyn kinase and BCR-ABL, which are implicated in chronic myelogenous leukemia, have higher activity in imatinib-resistant cells than in drug-susceptible cells [75-76], and single-cell assays detect this difference, even when resistant cells represent $<1 \%$ of the total population [76].

\section{Acknowledgments}

This work was supported by grants from the National Institutes of Health: Minority Opportunities in Research division of the National Institute of General Medical Sciences (NIGMS) grant \#K12GM000678 (MLK) and EB11763 and CA139599 (NLA).

\section{Glossary}

\author{
Amperometry \\ Capillary \\ electrophoresis \\ (CE)
}

Flow cytometry

Fluorogenic
substrate

FRET substrate

Indicator

Laser-induced

fluorescence (LIF)
An electrochemical detection method in which potential is fixed and current is measured as a function of time; for electroactive substrates and products, amperometry is an alternative to fluorescence detection

A separation technique based on migration of analytes in an electric field; in single-cell assays, enzyme isoforms or reactants and products of an enzymatic reaction (Figure 4c) may be separated prior to detection, allowing multiple enzymes to be assayed without spectrally-separated fluorescent substrates

A high-throughput technique in which individual cells are flowed past optical detectors; for enzyme assays, cells are loaded with a fluorogenic substrate and its enzymatic conversion to fluorescent product is measured (Figure 4a)

A substrate that is converted into a fluorescent product by an enzyme-catalyzed reaction

A substrate that undergoes a change in the efficiency of fluorescence resonance energy transfer (FRET) due to cleavage or conformational change when acted upon by an enzyme (Figure 3a)

Molecule(s) that responds to changes in local environment (e.g. $\mathrm{Ca}^{2+}$ concentration) or chemical state (e.g. phosphorylation or protein binding) with a change in some detectable property (e.g. fluorescence)

A detection method in which a photon from a laser beam electronically excites an atom or molecule and a fraction of the absorbed energy is then re-emitted as a photon; use of high 


\section{Microfabrication}

Peak capacity

Reporter

Scanning electrochemical microscopy (SECM) intensity laser illumination achieves lower detection limits than could be obtained with an arc lamp

A suite of techniques derived from the microelectronics industry for production of micron-sized structures, such as microwells (Figure $3 \mathrm{~b}$ ) and cell traps, which allow dense arrays of isolated cells to be processed and interrogated

The maximum number of peaks that can be separated with a resolution of 1 within a given separation space

A fluorescently tagged substrate that is processed by one or more enzymes and can be separated from its reacted form and detected

A scanning probe technique that produces an image based on current at an ultramicroelectrode tip; in single-cell enzyme assays, current is produced by oxidation or reduction of an electroactive product diffusing from a perforated cell (Figure 3c)

\section{References}

1. Altschuler SJ, Wu LF. Cellular heterogeneity: do differences make a difference? Cell. 2010; 141:559-563. [PubMed: 20478246]

2. Spiller DG, et al. Measurement of single-cell dynamics. Nature. 2010; 465:736-745. [PubMed: 20535203]

3. Tawfik DS. Messy biology and the origins of evolutionary innovations. Nat. Chem. Biol. 2010; 6:692-696. [PubMed: 20852602]

4. Zernicka-Goetz M, et al. Making a firm decision: multifaceted regulation of cell fate in the early mouse embryo. Nat. Rev. Genet. 2009; 10:467-477. [PubMed: 19536196]

5. Prussin C, et al. $\mathrm{T}_{\mathrm{H}} 2$ heterogeneity: Does function follow form? J. Allergy Clin. Immunol. 2010; 126:1094-1098. [PubMed: 20951419]

6. Lopez-Vergès S, et al. CD57 defines a functionally distinct population of mature NK cells in the human CH56 ${ }^{\text {dim }}$ CD16+ NK-cell subset. Blood. 2010; 116:3865-3874. [PubMed: 20733159]

7. Cohen AA, et al. Dynamic proteomics of individual cancer cells in response to a drug. Science. 2008; 322:1511-1516. [PubMed: 19023046]

8. Gascoigne KE, Taylor SS. Cancer cells display profound intra- and interline variation in following prolonged exposure to antimitotic drugs. Cancer Cell. 2008; 14:111-122. [PubMed: 18656424]

9. Arriaga EA. Determining biological noise via single cell analysis. Anal. Bioanal. Chem. 2009; 393:73-80. [PubMed: 18958456]

10. Elowitz MB, et al. Stochastic gene expression in a single cell. Science. 2002; 297:1183-1186. [PubMed: 12183631]

11. Taniguchi Y, et al. Quantifying E. coli proteome and transcriptome with single-molecule sensitivity in single cells. Science. 2010; 329

12. Newman JRS, et al. Single-cell proteomic analysis of $S$. cerevisiae reveals the architecture of biological noise. Nature. 2006; 441:840-846. [PubMed: 16699522]

13. Losick R, Desplan C. Stochasticity and cell fate. Science. 2008; 320:65-68. [PubMed: 18388284]

14. Raser JM, O'Shea EK. Control of stochasticity in eukaryotic gene expression. Science. 2004; 304:1181-1814.

15. Ozbudak EM, et al. Regulation of noise in the expression of a single gene. Nat. Genet. 2002; 31:69-73. [PubMed: 11967532]

16. Krutzik PO, et al. High-content single-cell drug screening with phosphospecific flow cytometry. Nat. Chem. Biol. 2008; 4:132-142. [PubMed: 18157122]

17. Sun X, Jin W. Catalysis-electrochemical determination of zeptomole enzyme and its application for single-cell analysis. Anal. Chem. 2003; 75:6050-6055. [PubMed: 14615980] 
18. Yashphe J, Halvorson HO. $\beta$-D-galactosidase activity in single yeast cells during cell cycle of Saccharomyces lactis. Science. 1976; 191:1283-1284. [PubMed: 1257751]

19. Invitrogen. The Molecular Probes handbook: A guide to fluorescent probes and labeling technologies. 11th edn. Invitrogen; 2010.

20. Ni Q, et al. Analyzing protein kinase dynamics in living cells with FRET reporters. Methods. 2006; 40:279-286. [PubMed: 16908183]

21. Kiyokawa E, et al. Fluorescence (Förster) resonance energy transfer imaging of oncogene actvitiy in living cells. Cancer Sci. 2006; 97:8-15. [PubMed: 16367915]

22. Frommer WB, et al. Genetically encoded biosensors based on engineered fluorescent proteins. Chem. Soc. Rev. 2009; 28:2833-2841. [PubMed: 19771330]

23. Ouyang M, et al. Simultaneous visualization of protumorigenic Src and MT1-MMP activities with fluorescence resonance energy transfer. Cancer Res. 2010; 70:2204-2012. [PubMed: 20197470]

24. Sharma V, et al. Peptide-based fluorescent sensors of protein kinase activity: design and applications. Biochim. Biophys. Acta. 2008; 1784:94-99. [PubMed: 17881302]

25. Kasili PM, et al. Optical sensor for the detection of caspase-9 activity in a single cell. J. Am. Chem. Soc. 2004; 126:2799-2806. [PubMed: 14995197]

26. Jiang T, et al. Tumor imaging by means of proteolytic activation of cell-penetrating peptides. Proc. Nat. Acad. Sci. 2004; 101:17867-17872. [PubMed: 15601762]

27. Hobson JP, et al. Imaging specific cell-surface proteolytic activity in single living cells. Nat. Methods. 2006; 3:259-261. [PubMed: 16554829]

28. Werner JM, Steinfelder HJ. A microscopic technique to study kinetics and concentration-response of drug-induced caspase-3 activation on a single cell level. J. Pharmacol. Toxicol. 2008; 57:131137.

29. Wudl L, Paigen K. Enzyme measurements on single cells. Science. 1974; 184:992-994. [PubMed: 4826171]

30. He M, et al. Selective encapsulation of single cells and subcellular organelles into picoliter- and femtoliter-volume droplets. Anal. Chem. 2005; 77:1539-1544. [PubMed: 15762555]

31. Baret J-C, et al. Fluorescence-activated droplet sorting (FADS): efficient microfluidic cell sorting based on enzymatic activity. Lab Chip. 2009; 9:1850-1858. [PubMed: 19532959]

32. Madou, M. Fundamentals of Microfabrication. 2nd edn. Vol. 1. CRC Press; 2002.

33. El-Ali J, et al. Cells on chips. Nature. 2006; 442:403-411. [PubMed: 16871208]

34. Di Carlo D, et al. Single-cell enzyme concentrations, kinetics, and inhibition analysis using highdensity hydrodynamic cell isolation arrays. Anal. Chem. 2006; 78:4925-4930. [PubMed: 16841912]

35. Sasuga Y, et al. Single-cell chemical lysis method for analyses of intracellular molecules using an array of picoliter-scale microwells. Anal. Chem. 2008; 80:9141-9149. [PubMed: 19551983]

36. Zhang X, et al. Quantitative determination of enzyme activity in single cells by scanning microelectrode coupled with a nitrocellulose film-covered microreactor by means of a scanning electrochemcial microscope. Anal. Chem. 2007; 79:1256-1261. [PubMed: 17263362]

37. Gao N, et al. Scanning electrochemical microscopy coupled with intracellular standard addition method for quantification of enzyme activity in single intact cells. Analyst. 2007; 132:1139-1146. [PubMed: 17955148]

38. Campbell RE. Fluorescent-protein-based biosensors: modulation of energy transfer as a design principle. Anal. Chem. 2009; 81:5972-5979. [PubMed: 19552419]

39. Honda A, et al. Spatiotemporal dynamics of guanosine 3',5'-cyclic monophosphate revealed by a genetically encoded, fluorescent indicator. Proc. Nat. Acad. Sci. 2001; 98:2437-2442. [PubMed: 11226257]

40. Green HM, Alberola-Ila J. Development of ERK activity sensor, an in vitro, FRET-based sensor of extracellular regulated kinase activity. BMC Chem. Biol. 2005; 5:1-8. [PubMed: 15998468]

41. O'Connor J-E, et al. The relevance of flow cytometry for biochemical analysis. IUBMB Life. 2001; 51:231-239. [PubMed: 11569917]

42. Shapiro, HM. Practical flow cytometry. 4th edn. Wiley-Liss; 2003. 
43. Dolbeare F. Dynamic assay of enzyme activities in single cells by flow cytometry. J. Histochem. Cytochem. 1979; 27:1644-1646. [PubMed: 230257]

44. Watson JV. Enzyme kinetic studies in cell populations using fluorogenic substrates and flow cytometric techniques. Cytom. 1980; 1:143-151.

45. Severin E, Seidler E. Flow cytometric assay of cytochemically demonstrated NAD(P)H oxidoreductase (diaphorase) activities. J. Histochem. Cytochem. 1998; 46:761-765. [PubMed: 9603788]

46. Telford WG, et al. Detection of endogenous alkaline phosphatase activity in intact cells by flow cytometry using the fluorogenic ELF-97 phosphatase substrate. Cytom. 1999; 37:314-319.

47. Berger J, et al. Glucocerebrosidase deficiency dramatically impairs human bone marrow haematopoiesis in an in vitro model of Gaucher disease. Brit. J. Haematol. 2010; 150:93-101. [PubMed: 20507316]

48. Gao N, et al. High-throughput single-cell analysis for enzyme activity without cytolysis. Anal. Chem. 2006; 78:3213-3220. [PubMed: 16643017]

49. Haddad, PR.; Robards, K. Inorganic species.. In: Heftmann, E., editor. Chromatography : fundamentals and applications of chromatography and related differential migration methods. 6th edn. Elsevier; 2004. p. 1156

50. Perfetto SP, et al. Seventeen-colour flow cytometry: unravelling the immune system. Nat. Rev. Immunol. 2004; 4:648-655. [PubMed: 15286731]

51. Xue Q, Yeung ES. Variability in intracellular lactate dehydrogenase isoenzymes in single human erythrocytes. Anal. Chem. 1994; 66:1175-1178. [PubMed: 8160961]

52. Sun X, et al. Measurement of alkaline phosphatase isoenzymes in individual mouse bone marrow fibroblast cells based on capillary electrophoresis with on-capillary enzyme-catalyzed reaction and electrochemical detection. Electrophoresis. 2004; 25:1860-1866. [PubMed: 15213985]

53. Brown RB, Audet J. Current techniques for single-cell lysis. J. R. Soc. Interface. 2008; 5:S131S138. [PubMed: 18426769]

54. Luzzi V, et al. Localized sampling of cytoplasm for Xenopus oocytes for capillary electrophoresis. Anal. Chem. 1997; 69:4761-4767. [PubMed: 9406526]

55. Shoemaker GK, et al. Multiple sampling single-cell enzyme assays using CE-laser-induced fluorescence to monitor reaction progress. Anal. Chem. 2005; 77:3132-3137. [PubMed: 15889901]

56. Meredith GD, et al. Measurement of kinase activation in single mammalian cells. Nat. Biotech. 2000; 18:309-312.

57. Jiang D, et al. Single-cell analysis of phosphoinositide 3-kinase (PI3K) and phosphatase and tensin homolog (PTEN) activation. Faraday Discuss. 2010 DOI: 10.1039/C005362G.

58. Krylov SN, et al. Correlating cell cycle with metabolism in single cells: combination of image and metabolic cytometry. Cytom. 1999; 37:14-20.

59. Le XC, et al. Single cell studies of enzymatic hydrolysis of a tetramethylrhodamine labeled triglucoside in yeast. Glycobiol. 1999; 9:219-225.

60. Nelson AR, et al. Myristoyl-based transport of peptides into living cells. Biochem. 2007; 46:14771-14781. [PubMed: 18044965]

61. Zarrine-Afsar A, Krylov SN. Use of capillary electrophoresis and endogenous fluorescent substrate to monitor intracellular activation of protein kinase A. Anal. Chem. 2003; 75:3720-3724. [PubMed: 14572035]

62. Marc PJ, et al. Coaxial flow system for chemical cytometry. Anal. Chem. 2007; 79:9054-9059. [PubMed: 17979298]

63. Jiang D, et al. Microelectrophoresis platform for fast serial analysis of single cells. Electrophoresis. 2010; 31:2558-2565. [PubMed: 20603824]

64. Sims CE, Allbritton NL. Analysis of single mammalian cells on-chip. Lab Chip. 2007; 7:423-440. [PubMed: 17389958]

65. Zare RN, Kim S. Microfluidic platforms for single-cell analysis. Annu. Rev. Biomed. Eng. 2010; 12:187-201. [PubMed: 20433347] 
66. Chang A, et al. BRENDA, AMENDA, and FRENDA the enzyme information system: new content and tools in 2009. Nucleic Acid Res. 2009; 37:D588-D592. [PubMed: 18984617]

67. Arkhipov SN, et al. Chemical cytometry for monitoring metabolism of a Rasmimicking substrate in single cells. Cytom. Part A. 2005; 63A:41-47.

68. Mann M, Jensen ON. Proteomic analysis of post-translational modifications. Nat. Biotech. 2003; 21:255-261.

69. Wang D, Bodovitz S. Single cell analysis: the new frontier in '-omics'. Trends Biotechnol. 2010; 28:281-290. [PubMed: 20434785]

70. Varki, A., editor. Essentials of glycobiology. Cold Spring Harbor Laboratory Press; 1999.

71. Zheng XT, et al. Optical detection of single cell lactate release for cancer metabolic analysis. Anal. Chem. 2010; 82:5082-5087. [PubMed: 20469833]

72. Agnes RS, et al. Suborganelle sensing of mitochondrial cAMP-dependent protein kinase activity. J. Am. Chem. Soc. 2010; 132:6075-6080. [PubMed: 20380406]

73. Rehm M, et al. Single-cell fluorescence resonance energy transfer analysis demonstrates that caspase activation during apoptosis is a rapid process. J. Biol. Chem. 2002; 277:24506-24514. [PubMed: 11964393]

74. Whitmore CD, et al. Metabolic cytometry. Glycosphingolipid metabolism in single cells. Anal. Chem. 2007; 79:5139-5142. [PubMed: 17567107]

75. Wang Q, et al. Multicolor monitoring of dysregulated protein kinases in chronic myelogenous leukemia. ACS Chem. Biol. 2010; 5:887-895. [PubMed: 20583816]

76. Mizutani T, et al. A novel FRET-based biosensor for the measurement of BCRABL activity and its response to drugs in living cells. Clin. Cancer Res. 2010; 16:3964-3975. [PubMed: 20670950]

77. Oh-hora O. Calcium signaling in the development and function of T-lineage cells. Immunol. Rev. 2009; 231:210-224. [PubMed: 19754899]

78. Ross PE, et al. Dynamics of ATP-induced calcium signaling in single mouse thymocytes. J. Cell Biol. 1997; 138:987-998. [PubMed: 9281578]

79. Colman-Lerner A, et al. Regulated cell-to-cell variation in a cell-fate decision system. Nature. 2005; 437:699-706. [PubMed: 16170311]

80. Brown RB, et al. Single amino acid resolution of proteolytic fragments generated in individual cells. Cytom. Part A. 2010; 77A:347-355. 
(a)

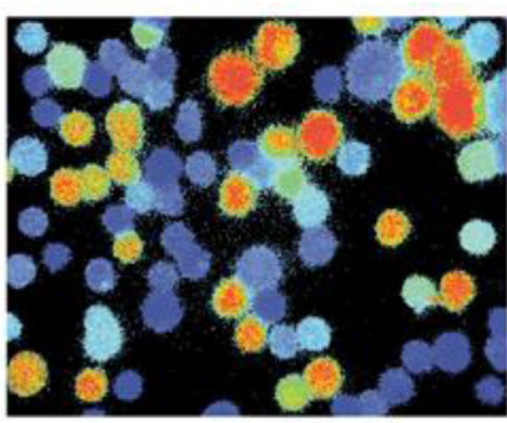

ATP-INDUCED CALCIUM SIGNAL

(c)

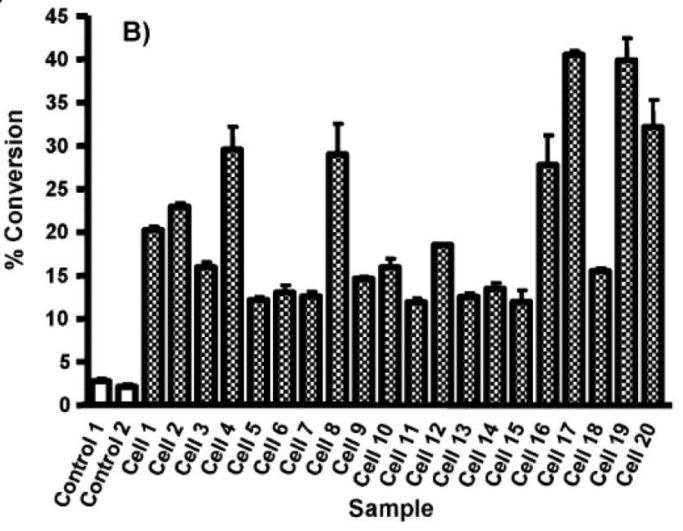

(b)

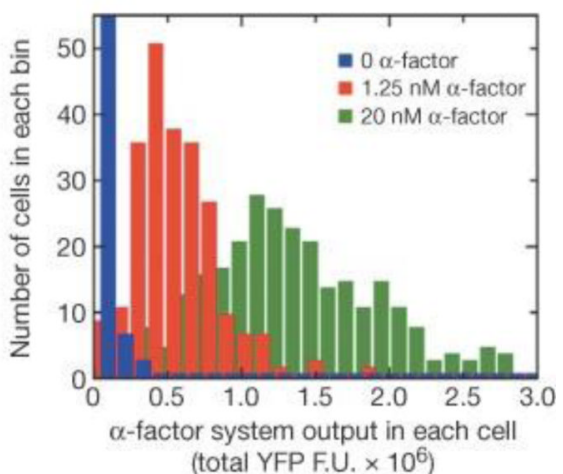

(d)

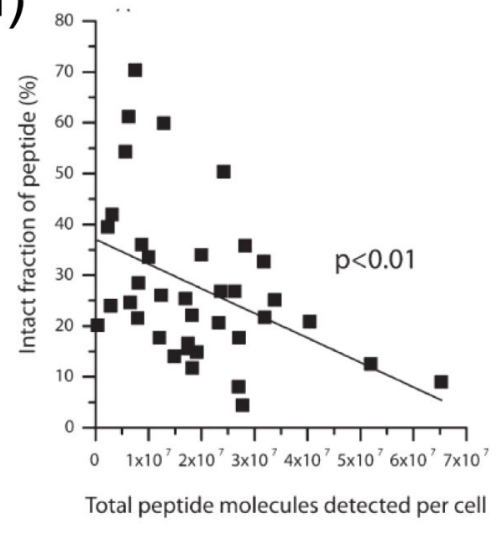

Figure 1.

Examples of pathway- and enzyme-level cellular heterogeneity. (a) ATP-induced calcium signaling in mouse thymocytes results from a multi-step process in which the release of intracellular calcium stores triggers calcium influx through $\mathrm{Ca}^{2+}$ release-activated $\mathrm{Ca}^{2+}$ (CRAC) channels [77]. The cells were all stimulated with the same ATP concentration, but variability in this pathway results in dramatic cell-to-cell differences in calcium influx. The heat map indicates intracellular $\mathrm{Ca}^{2+}$ concentration (blue: $0 \mu \mathrm{M}$, red: $1.0 \mu \mathrm{M}$ ). Reproduced with permission from [78]. (b) Mating events in yeast are initiated by a complex response to the pheromone $\alpha$-factor that involves scaffold proteins, enzymes, and transcription factors. Mating response varies widely between cells stimulated with the same $\alpha$-factor concentration. Reprinted with permission from [79]. (c) Directed single-cell enzyme assays probe the activities of enzymes that contribute to complex cell functions. These assays might examine a specific enzyme activity, such as $\alpha$-glucosidase II-catalyzed conversion of a rhodamine-labeled disaccharide to monosaccharide in Sf9 cells (reprinted with permission from [55], or (d) the total enzymatic activity of a broad class of enzymes, such as the net proteolytic activity of individual TF-1 cells toward $\beta$-amyloid precursor protein ( $\beta$-APP). Each data point represents one cell. Reprinted with permission from [80]. 


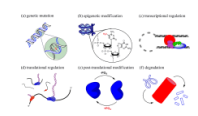

Figure 2.

Origins of cellular heterogeneity in enzyme activity. The sources of heterogeneity are diverse and complex, including variation at both the nucleic acid (a-c) and protein (d-f) levels of gene expression. These biomolecular events affect enzyme activity by changing the chemical identity $(\mathrm{a}, \mathrm{e})$ and concentration (b-d, f) of an enzyme within a single cell. In each schematic, the biochemical source of variability is highlighted in red. (a) Genetic mutations change the corresponding amino acid sequence and consequently alter the activity of the gene product. (b) Epigenetic modifications, including DNA methylation, are implicated in varying levels of gene expression. (c) Activator and repressor proteins interact with eukaryotic transcription machinery to up-regulate or down-regulate, respectively, the transcription of specific genes. (d) Translational regulation, including variation in the initiation rate of translation events, affects the production of protein from mRNA transcripts. (e) Post-translation modifications, including phosphorylation, commonly regulate enzyme activity and binding partners through changes in conformation, hydrophobicity, and/or charge. (f) Rates of degradation by the proteasome (pictured) and proteases influence the concentration and lifetime of enzymes in a cell. 

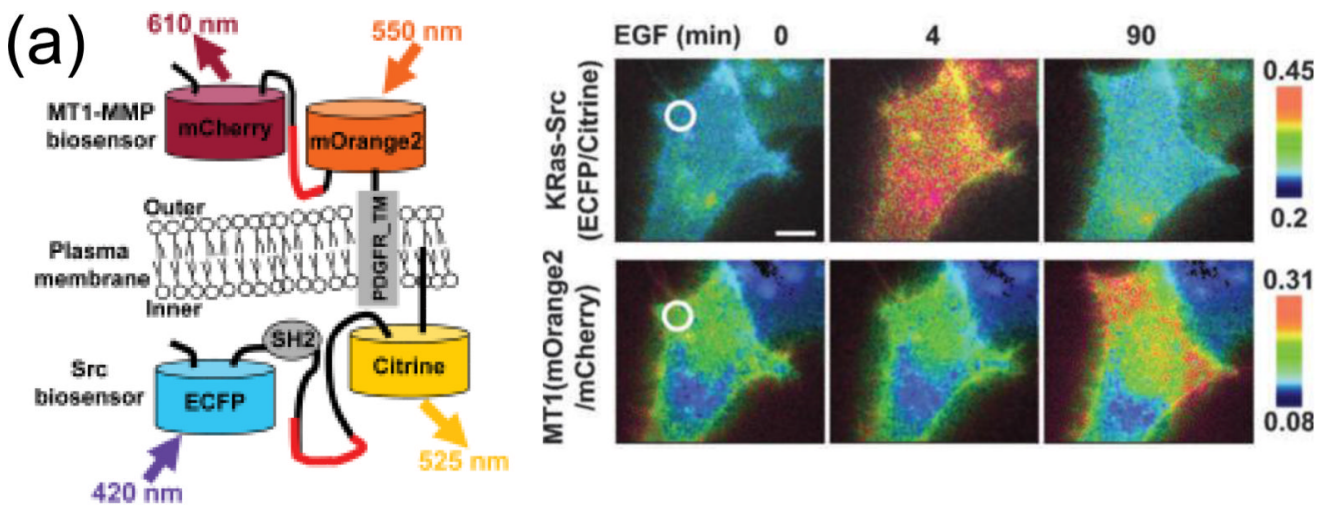

(b)

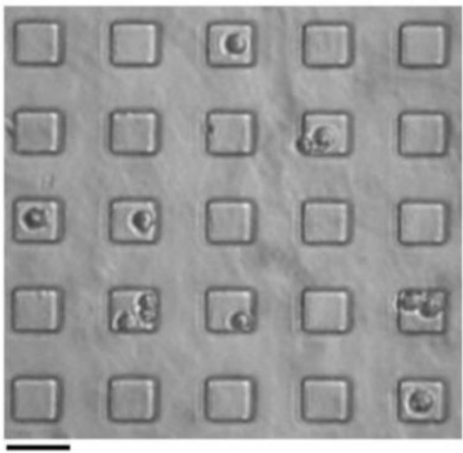

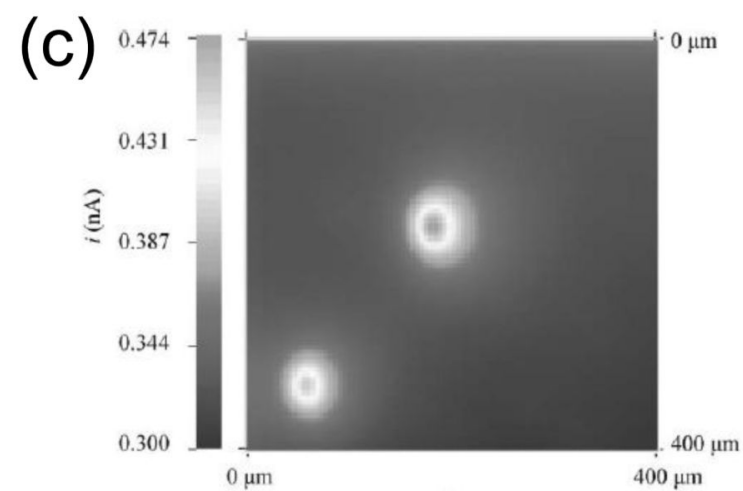

Figure 3.

Image-based methods for single-cell enzyme assays. (a) Two FRET pairs [enhanced cyanine fluorescent protein (ECFP) paired with citrine and mOrange paired with mCherry] permit simultaneous measurements of Src kinase and MT1-MMP as a function of time after exposure to EGF. The heat maps show the FRET ratio for each pair and reflect enzyme activity. Scale bar, $30 \mu \mathrm{m}$. The white circles are pertinent to another panel of the original figure which was not reproduced here. Reprinted with permission from [23]. (b)

Microfabricated cell arrays trap individual cells and lysates at high density in known locations. Scale bar, $50 \mu \mathrm{m}$. Reprinted with permission from [35]. (c) SECM shows benzoquinone production from the peroxidase-catalyzed reaction of hydroquinone and hydrogen peroxide. Current $(i)$ from reduction of benzoquinone is directly related to peroxidase activity in the two perforated cells. Reproduced with permission from [37]. 


\section{(a)}

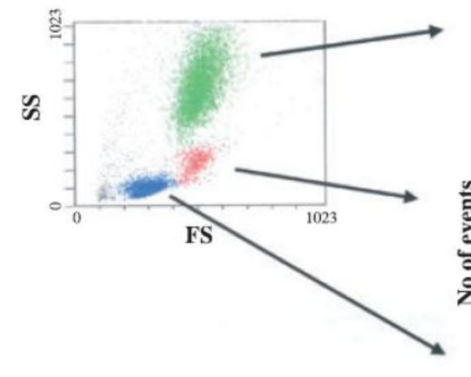

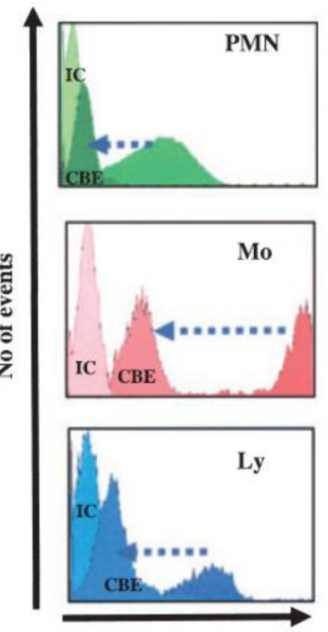

FITC (b)
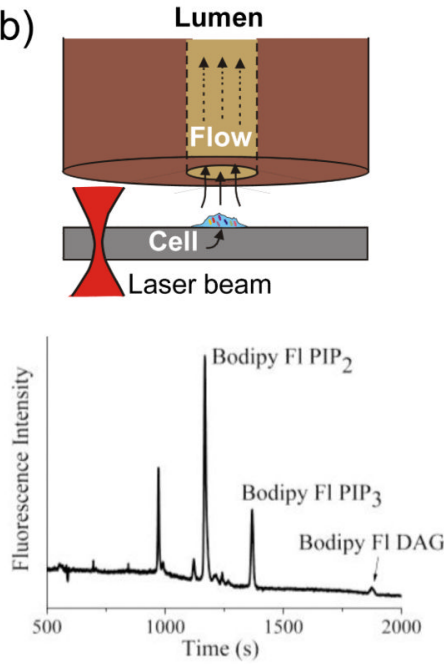

Figure 4.

Flow- and separation-based methods for single-cell enzyme assays. (a) Flow cytometry data on glucocerebrosidase activity in polymorphonuclear cells (PMN), monocytes (Mo) and lymphocytes (Ly). Cell types are determined by forward (FS) and side scatter (SS), and enzyme activity by fluorescence signal (FITC) from cleavage of a fluorogenic substrate. Blue arrows indicate the change in activity upon treatment with the inhibitor conduritol B epoxide (CBE), and signals for an unspecific isotypic control (IC) are shown for reference. Reprinted with permission from [47]. (b) An alternative capillary electrophoresis technique separates fluorescent reporters from lysed cells. PI3K and phospholipase $\mathrm{C}$ in rat basophilic leukemia cells convert a Bodipy-fluorescein-labeled reporter, phosphatidyl-inositol 4,5bisphosphate (Bodipy Fl PIP 2 ), into Bodipy Fl PIP 3 and Bodipy Fl diacylglycerol (DAG). Reproduced with permission from [57]. 
(a)

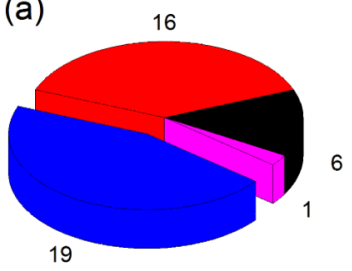

(c)

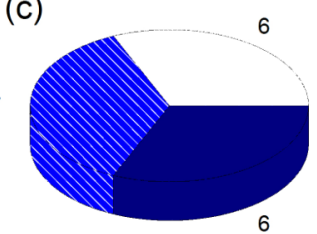

(b)

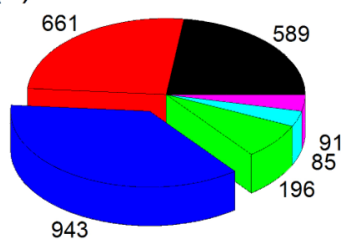

(d)

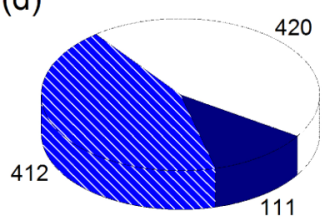

EC1: oxidoreductases (e.g., dehydrogenase)

EC2: transferases (e.g., kinase)

EC3: hydrolases (e.g., peptidase)

EC4: lyases (e.g, decarboxylase)

EC5: isomerases (e.g., mutase)

EC6: ligases (e.g., synthetase)

\section{Figure 5.}

Comparison of the distribution of enzymatic reactions with published single-cell assays to those known in humans. (a) Enzymatic reactions for which single-cell activity assays have been published, and (b) the total number of enzyme-catalyzed reactions in humans from the BRENDA are grouped by their top-level EC number. $(\mathrm{c}, \mathrm{d})$ Break-out pie charts of hydrolase (EC 3) reactions with (c) published single-cell activity assays compared to (d) all human hydrolases reactions in BRENDA. Although every published single-cell enzyme assay might not be included, the data shown here are reasonably complete and representative.

Additionally, because EC numbers technically correspond to a reaction rather than to a specific enzyme molecule, two or more closely related enzymes may share a single EC number. Overall, glycosylases are notably overrepresented owing to the ready availability of fluorogenic substrates for these enzymes, whereas oxidoreductases, lyases and isomerases are underrepresented. 